\title{
Preionization Studies in ZT-40M
}

R. G. Watt

J. N. Downing

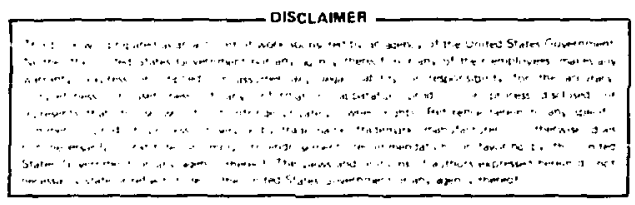


PREIONIZATIUN STUDIES IN ZT-4OM

by

R. G. Watt and J. N. Downing

\begin{abstract}
'
A study of preionization techniques has been performed in 2T-40M at Los Alamos National Laboratory. The results of this study and the techniques routinely utilized to prelonize the plasma in this device are presented. A simple theoretical model involving drift motion of the electrons in the inhomogeneous torofdal field $B_{\text {p }}$ present during breakdown is examined. This model is shown to offer a conceptual explanation for the breakdown 1 imits in $\mathrm{ZT}-40 \mathrm{M}$. Thls simple theory appears to Indicate that the preionization system produces photoelectrons locaily at the wall as opposed to acting as a volume source of electrons.
\end{abstract}

\title{
I. INTRODUCTION
}

In the present generation of metallic vacuum vessel Reverse Field Pinches (RFP's), the behavior of the plasma current decay ( $\dot{I}_{\phi}$ ) as a function of $f 111$ pressure $\left(\mathrm{P}_{0}\right)$ may be used as an Indicator of the best operating regime, $A$ study in the ZT-40M device at Los Alamos National Laboratory of the behavior of $\dot{I}_{\phi}$ vs $P_{0}$ has been an integral component In the definition of the proper f 111 pressure for maximum plasma temperature and lifetime. To cover the possible operating regimes completely so that one does not miss the best operating point, it is necessary to create the plasma with fill pressuras as low as 1 mtorr. With a 250 us $I_{\phi}$ rlsetime, pressures below 1.8 mtorr are Inaccessible without creating a prelonized plasma prior to application of the toroldal voltage $\left(E_{\phi}\right)$. At lower pressures, $E_{\phi} / P_{0}$ is too large for the breakdown to occur spontaneously and one nust ald the breakdown with a source of prefonization. This report will cover the techniques used routinely on 2T-40M for this purpose, as well as cther techniques investigated. A simple 
theoretical model will be presented in an effort to explain the capabilities and IImitations of the present approach to preionization in ZT-40M.

\section{APPARATUS}

A commonly used technique for creating a low level of preicnization in a plasma containment device is the exposure of the neutral fill gas ( $D_{2}$ in $\mathrm{ZT}-40 \mathrm{M}$ ) to an intense source of ultraviolet radiation. On $\mathrm{ZT}-40 \mathrm{M}$ this technique is used in a manner that is simple to accomplish and that uses Inexpensive, commerclally avallable light sources. A Vivitar 215 commercial electronic photoflash untt with its amber plastic filter removed is positioned to 1lluminate the plasma through a 2-1n.-diam. quartz window. The combination of a Xenon flash tube in the Vivitar unft and quartz window materiai permits the UV portion of the spectrum from the flash untt to reach the f111 gas with relatively 1ittle attenuation (transmission of the quartz is reasonebly flat between $200-700 \mathrm{~nm}$ ).

Such untts are used to prefonize some portion of the fill gas at up to four points toroidally around $\mathrm{ZT}-40 \mathrm{M}$. The units are all powered by a commori battery pack and triggered simultaneously by the thyristor circuit shown in Fig. 1. The trigger clrcult ts powered by the flash unit itself and is used

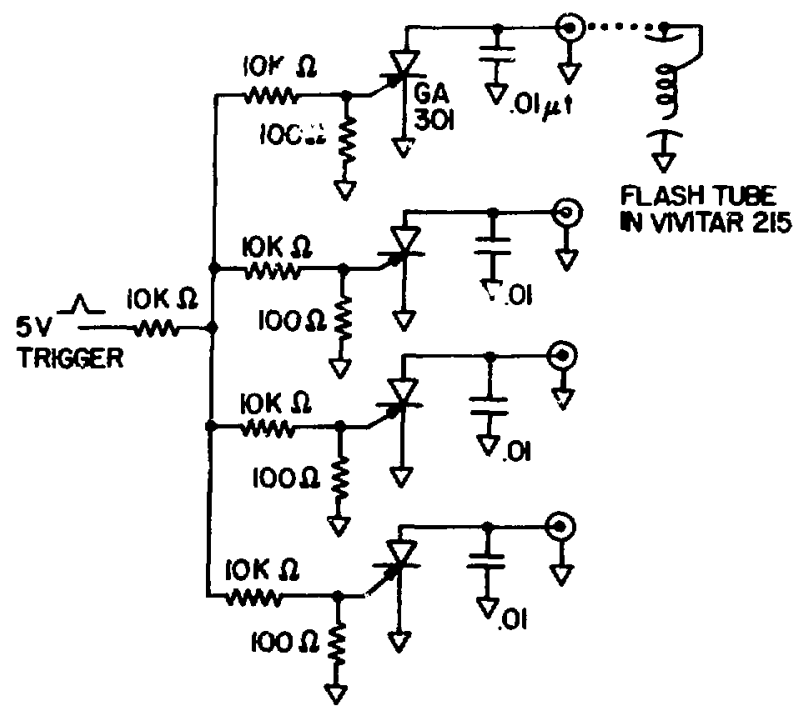

Fig. 1.

Thyristor clrcults used to actuate the Vivitar 215 strobe 1ights. 
to short one end of the flash tube and an assoclated tickler coli from about 8 n $V$ to ground, causing the tube to fire. The duration of the 11 ght pulse is approximately $0.5 \mathrm{~ms}$ and 18 positioned in time so that the application of the toroldal drive voltage $\left(E_{\phi}\right)$ occurs during the decay of the 11ght pulse. A typical time history of the UV portion of the pulse (viewed through a Corning 7-54 optical filter which trangmits light at wavelengths below $400 \mathrm{~nm}$ ) is shown In Fig. 2. The trigger circult and flash unit combination has a timing fitter of 1 ess than $10 \mathrm{\mu s}$. It was found experimentally that the flash units had a tendency to is by themselves when used with a trigger circuit contalning no bypass (storage) capacitor and a large gate resistor. This was presumably caused by nolse on the anode of the SCR, which coupled through the Miller capacitance to the gate and caused the unit to fire. Insertion of the anode capacitor and a low resietance shunt to ground on the gate eliminateo the problem resulting in the circult shown in Fig. 1.

This system, usually containing elther 3 or 4 photoflash units, is now used routinely every shot on $\mathrm{ZT}-40 \mathrm{M}$. The battery pack, consisting of two $2 \mathrm{~F} 2 \mathrm{H}$ Burgess 3-V batterles in parallel, has powered the system of four lamps for more than 1,000 shots and has fust recently been replaced. The effects of the

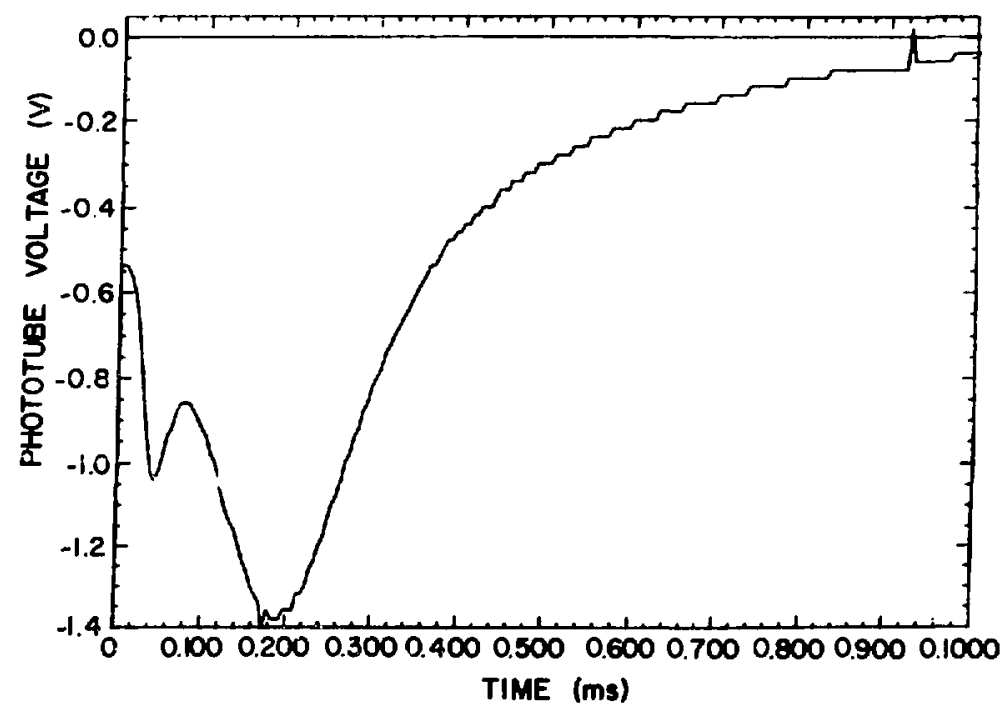

F18. 2.

Typical time history of a flash discharge (intensity increases downward). 
prefonization system on the breakdown limit (lowest attainable $P_{0}$ ) will be discussed in the next section.

In an effort to extend the breakdown limit below that obtainable with the four strobes, a second prelunization source was also experimented with. This second source was a nude high-vacuum lonization gauge inserted in one of the tubulations connected to $\mathrm{ZT}-40 \mathrm{M}^{\prime}$ s metal liner. The purpose of the structure was to replace the electrons created using photolonization from one of the four strobes by electrons emitted thermionically from the hot filament of the ionization gauge. This source was capable of being blased up to $535 \mathrm{~V}$ negative with respect to the metal liner. This technique was used here in an attempt to gain access to the $\mathrm{f} 111$ pressure reglme below 1 ntorr. The results are discussed in the next section, but in general at morerate bias voltages ( $180 \mathrm{~V}$ ) the results, using this source in conjunction with three strobes, were similar to those obtained with four photolonization sources.

\section{EXPERIMENTAL RESULTS}

The breakdown process in the plasma under the influence of the applied field $E_{\phi}$ takes a finite amount of time. In 2T-40M at high pressures ( $>3$ mtorr), the breakdown takes a minimum of 15-20 us. This delay between application of $\vec{E}_{\phi}$ and the beginning of the toroldal current in the plasma is directly observable as a momentary hesitation in the current rise, as viewed by a Rogowski probe viewing $\left\langle B_{\theta}\right\rangle$ at the wall outside the liner. For the first 15-20 us, all the current is carried in the 11ner ( $30 \mathrm{kA}$ at $15 \mathrm{kV}$ charge on the $E_{\phi}$ bank). At later times ( $20 \mu \mathrm{s}$ ) the plasma begins to conduct and the current rises sharply to the 200-kA level in $250 \mathrm{\mu s}$. The delay between the I Iner current and plasma current is clearly seen 1 the trace of $I_{\phi}$ shown in Fig. 3. To quantize the preionization prccess, this time delay was measured at constant $E_{\phi}$ for a variety of fill pressures and several different source configurations. As one approaches the limiting value of $P_{0}$, below which breakdom will fall, this delay, in the space of approximately 0.1 mtorr, will go from low values (15-25 $\mu s)$ to the limiting value allowed by the

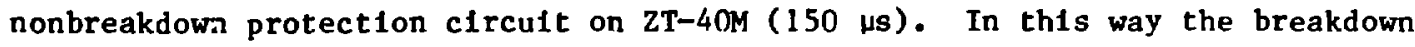
IImit may be established as that fill pressure at which the lonization lag time has increased to values above 40-50 $\mu \mathrm{s}$. 


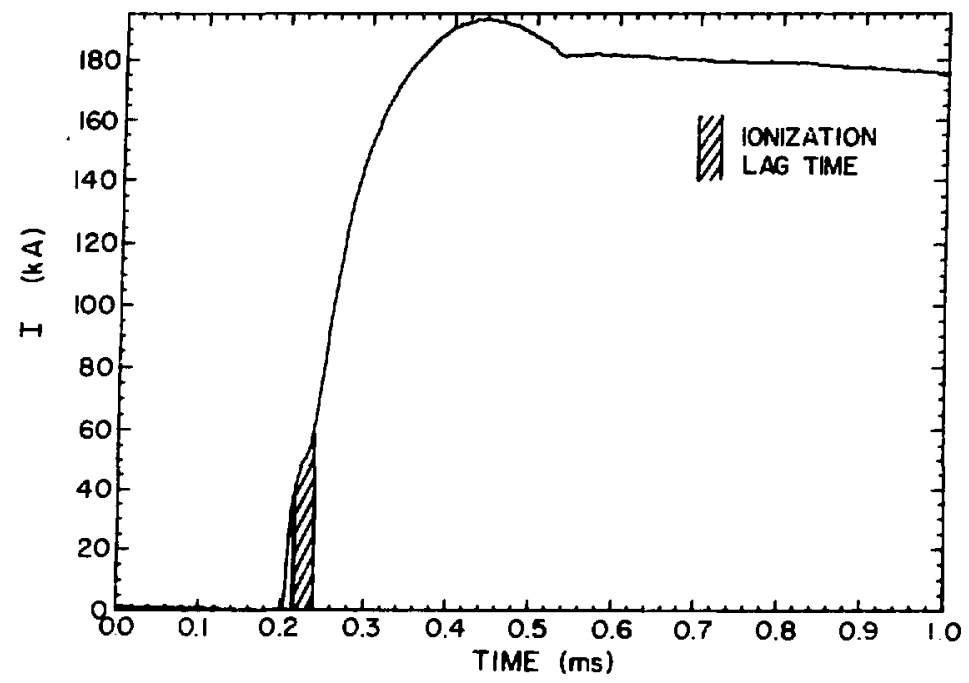

F1g. 3.

Typical plasma current inttiation showing ion: ation lag time (shaded portion).

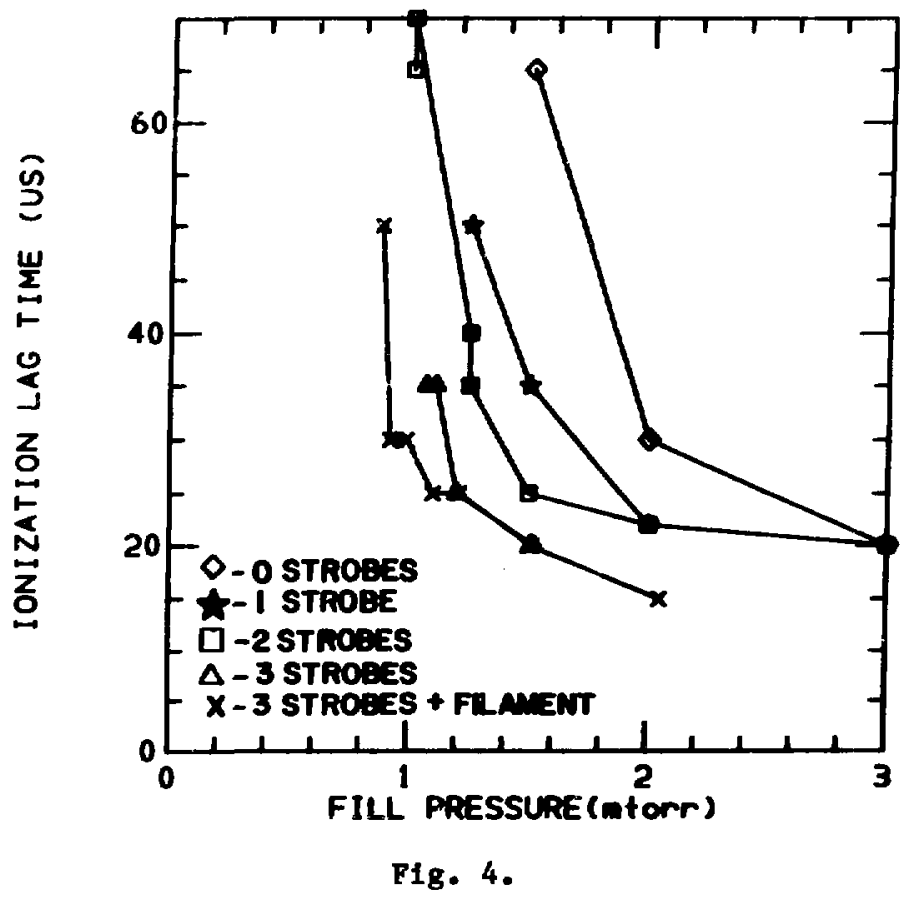

Ionization lag times measured in $2 T-40 \mathrm{M}$ as a function of initial fill pressure. 
Measurements of the ionization $\mathrm{lag}$ time are shown in Fig. 4. In order to present the data in the clearest possible manner, only the data with one to three strobes positioned at $0^{\circ}, 90^{\circ}$, and $180^{\circ}$ from the north pump stand and the best results with three strobes and the filament (blas at $-180 \mathrm{~V}$ ) are shown. Other data with the filament produced breakdown limits at higher $P_{0}$ levels, presumably because with no blas voltage or with too large blas voltage $(-535 \mathrm{~V})$, the thermionic electrons do not get out into the breakdown volume. The lower limit with four sources is in the vicinity of 1 mtorr regardless of the source type. It is belleved that this is an effect of the position of the nearest neighbor source and that the only improvement will come from much larger numbers of sources distributed evenly throughout the machine.

IV. THEORY

In an effort to understand the limits and capabilicles of the breakdown in ZT-40M, an extremely simplified model may be invoked. This theory must be composed of two parts, an examination of the local breakdown physics and an examination of the process throughout the entire torus. In an effort to provide a complete theoretical package in one location, both aspects of the nroblem are discussed here in some detail. The two aspects of the problem will be considered in sequence.

The problem is that of a neutral gas background in which low ionization levels are created by the applied $E_{\phi^{*}}$ Because during the breakdown phase the cuirent $I_{\phi}$ in the plasma is small but the toroidal field $B_{\phi}$ is large, the plasma created by the breakdown process exists in a simple torus that 1s incapable of sustaining an MHD or single-particle equilibrium. ${ }^{2}$ The loss of equllibrium is caused by the drift motion of the charged particles due to the curvature (drift velocity proportional to $v_{p}^{2}$ ) and gradient (drift velocity proportional to $v_{1}^{2}$ ) of the toroldal fleld.

The problem of loss of equilibrium of a motionless, thermal plasma due to drift motion in the presence of a uniform magnetic fleld under the force of gravity has been solved by Longmire. ${ }^{3}$ With replacement of the gravitational force $g$ In this analysis by the centrifugal force $v^{2} / R$ the same solution may be made to apply to the loss of equilibrium of a plasma in a torus in the absence of an external accelerating toroldal electric foeld. ${ }^{3}$ Hirano $^{4}$ has used this solution with ion temperatures estimated frjm measured $x$-ray spectra 
during breakdown to estimate the drift velocity in a fast risetime, sma11 cross-section torus.

In the presenre of a toroldal electric field, the problem changes because the electrons become the dominant species. Longmire's solution must be modified to reflect the fact that the velocity distribution functions (particularly for the electrons) now have a preferred direction and cannot be considered to be eyen remotely thermal. Such a modification is out 11 ned here. In the case of ZT-40M, the Initlal process of the Instability is caused by the acceleration of the created electrons along $E_{\phi}$, which results in a cross-field drift dominated by the curvature term for the electrons (rather than the lons as in Longmire's analysis) because $v_{1, e} \gg v_{1, e^{\prime}} v_{1,1}, v_{1,1}$. This drift is charge dependent and results in a charge separation and resulting vertical electric field $E_{z}$, which produces a drift in the major radial direction for al1 charged species. This latter drift is the dominant motion and results in rapld loss of plasma to the wall in a simple torus. In the following, a coordinate system in which $\phi$ is the toroldal coordinate, $\theta$ is a polotdal coordinate, and $z$ is the vertical coordinate is used. One may write down expressions for the dominant velocities in the problem ${ }^{5}$ given in MKS units.

Velocity due to the driving field $E_{\phi}$ :

$$
\vec{v}_{\|}=\frac{-q_{\alpha} \vec{E}_{\phi}}{m_{\alpha}} \tau_{1},
$$

where $\tau_{1}$ is the time between randomizing collisions, $q_{\alpha}$ is the charge of spectes $\alpha, m_{\alpha}$ is the mass of spectes $\alpha$, and $\vec{E}_{\phi}$ is the toroidal applied field.

Curvature drift velocity:

$$
\begin{aligned}
& \vec{v}_{D,(B \cdot \nabla) \vec{B}}=\frac{m_{\alpha}}{q_{\alpha}} v_{\|}{ }^{2} \frac{\vec{B} \times(\vec{B} \cdot \nabla) \vec{B}}{B^{4}} \\
& \left.\mid \vec{v}_{D,(B} \cdot \nabla\right) \vec{B} \mid \simeq \frac{m_{\alpha} v_{\|}^{2}}{q_{a}} \frac{1}{R_{\phi}} .
\end{aligned}
$$


$\vec{E} \times$ B drift velocity:

$$
\overrightarrow{\mathrm{D}}_{\mathrm{D}}, \overrightarrow{\mathrm{E}}_{z} \times \overrightarrow{\mathrm{B}}_{\phi}=\frac{\overrightarrow{\mathrm{E}}_{z} \times \overrightarrow{\mathrm{B}}_{\phi}}{\mathrm{B}_{\phi}^{2}},
$$

where the third expression is the approximate value of the curvature drift velocity for a simple torus of major radius $R$ and toroldal field $B_{\phi^{*}}$ The vertical electric field induced $\left(E_{z}\right)$ is given (for the one-dimensional problem with electron density $n_{e}$ ) by

$$
E_{z}=\frac{-n_{e} e^{\vec{z}}}{\varepsilon_{0}} \simeq \frac{-n_{e} e^{e}}{\varepsilon_{0}} \vec{v}_{z} \tau_{2} \text {, }
$$

where $\vec{v}_{z}$ is the velocity along the $\hat{z}$ direction $\left[\vec{v}_{D,}(\vec{B}, \nabla) \vec{B}\right]$ and $\tau_{2} 1 s$ the time during which $\vec{v}_{z}$ acts. In this case the $n_{2} 1 s$ that electron density generated locally caused by the breakdown process and thus is time dependent. The electron density at any time is created by the lonization process in the applied fleld, corrected by whatever fluld motion occurs (here caused by the drift motion induced by $B_{\phi}$ ). The controlling equation 18 the mass conservation equation of the two-fiuld MHD theory, neglecting cross-field diffusion,

$$
\frac{\partial n_{e}}{\partial t}+n_{e} \nabla \cdot \vec{u}=s(\vec{r}, t),
$$

where $S$ is the electron source term, here given by ${ }^{6}$

$$
S(r, t)=u \text { an } e
$$

and $\vec{u}$ is the electron fluid velocity. The coefficient $\alpha$ is the Townsend coefficlent given by the expression 


$$
\alpha=510 \mathrm{P}_{0} \text { (torr) exp }\left\{-1.388 \times 10^{4} \mathrm{P}_{\mathrm{o}}(\text { torr }) / \mathrm{E}_{\phi}(\mathrm{V} / \mathrm{m})\right]
$$

The lons travel with the electrons due to the Induced $E_{z} \times \vec{B}_{\phi}$ drift, which is mass independent, but contribute nothing (except for a collision term) to the breakdown process which involves only electron-inpact lonization of the neutral background. The electron-1on collision term, which does not appear explicitly in this simple model, does contribute to one of the time scales in the problem (the time $\tau_{1}$ to be defined later). Using the above drifts in

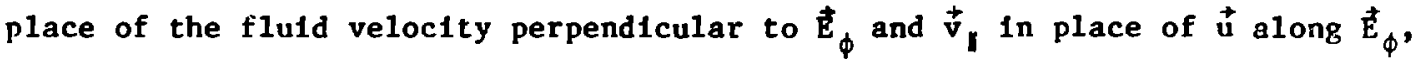
we may write the controling equation

$$
\frac{\partial n_{e}}{\partial t}=-v_{\|} \alpha n_{e}-n_{e} \nabla_{\perp} \cdot \vec{v}_{D}
$$

This equation could be solved numerically, along with the time-dependent momentum equation that generates the velocities, but such an analysis is beyond the scope of this report.

In keping with che simplistic model invoked here, let us define some average quantities $\left\langle v_{\|}\right\rangle,\left\langle v_{1}\right\rangle(\vec{B} \cdot \nabla) \vec{B},\left\langle E_{1}\right\rangle$, and $\left\langle v_{1}\right\rangle\left\langle E_{1}\right\rangle \times E_{\phi}$ in the following manner.

$$
\begin{aligned}
& \left\langle v_{h}\right\rangle=-\frac{e E_{\phi}}{m_{e}} \tau_{1}, \\
& \left.\left\langle v_{\perp}\right\rangle_{(\vec{B}} \cdot \nabla\right) \vec{B}=-\frac{m_{e}\left\langle v_{\|}\right\rangle^{2}}{e} \frac{1}{R B_{\phi}}, \\
& \left\langle F_{\perp}\right\rangle=-\frac{\text { en }}{\varepsilon_{0}} \tau_{2}\left\langle v_{\perp}\right\rangle(\vec{B} \cdot \nabla) \vec{B} \text {, and } \\
& \left\langle v_{\perp}\right\rangle_{\left\langle\vec{E}_{\perp}\right\rangle \times \vec{B}_{\phi}}=\frac{\left|\left\langle\vec{E}_{\perp}\right\rangle \times \vec{B}_{\phi}\right|}{B_{\phi}^{2}}=\frac{\left|\left\langle\vec{E}_{1}\right\rangle\right|}{\vec{B}_{\phi}} \cdot
\end{aligned}
$$


The times $\tau_{1}, \tau_{2}$ are characteristic times in the problem representing how long $E_{\phi}$ may accelerate electrons before a collision randomizes their directed velocity, and how long the $(\vec{B} \cdot \nabla) \vec{B}$ drift can act to create charge separation before saturation occurs, respectively. We may approximate the mass conservation equation (noting $\partial / \partial z \simeq 0$ ) by

$$
\frac{\partial n_{e}}{\partial t} \simeq-\left\langle v_{y}\right\rangle a n_{e}-\frac{n_{e}}{R}\left\langle v_{\perp}\right\rangle\left\langle\vec{E}_{\perp}\right\rangle \times \vec{B}_{\phi},
$$

which may be rewritten in general as

$$
\frac{\partial n_{e}}{\partial t}=\frac{e E_{\phi}}{m_{e}} \tau_{1} n_{e}\left\{\alpha-\frac{e n_{e}}{\varepsilon_{0}} \frac{1}{\left(R B_{\phi}\right)^{2}} E_{\phi} \tau_{1} \tau_{2}\right\} .
$$

Written in this manner, we may conclude that convection dominated breakdown cannot occur unless the right side is positive; that is, the breakdown occurs when

$$
\alpha>\frac{e n_{e}}{E_{0}} \frac{1}{\left(R B_{\phi}\right)^{2}} \cdot F_{\phi} \tau_{1} \tau_{2}
$$

The above equations represent the microscopic part of the breakdown picture and include the effects of the Townsend avalanche caused by $\mathrm{E}_{\phi}$ and the convection out of the region due to drifts. If this convection occurs faster than the Ionization, the process will quench locally and breakdown will not $\operatorname{occur}(\mathrm{Eq} \cdot$. 9).

There is also a second part to the process. Because the total current $I_{\phi}$ in the plasma must go completely around the torus, an electron cascade originating at one point in the torus must eventually reconnect with itself after a full toroldal revolution or with the source region of another electron cascade in the torus to make a complete charge-carrying ring. If this global 
cascade reconnection is defeated because the cascade from one region drifts out and contacts the wall before it can combine with other cascades to complete the ring of charge carriers, the breakdown, while occurring locally, will have difficulty sustaining 1tBelf. Th1s $11 \mathrm{mit}$ may also be expressed in terms of the drifts. If the major radial drift, acting over the time necessary for electrons to get from one source region to another, produces a larger major radial displacement than the diameter of the torus, no breakdown can occur. This process again is time dependent, but we may estimate the IImit in terms of average $\left\langle v_{D}\right\rangle$ and $\left\langle v_{\|}\right\rangle$as

$$
\left\langle v_{D}, E_{z} \times \vec{B}_{\phi}\right\rangle \cdot \frac{d}{\left\langle v_{\|}\right\rangle}<a,
$$

where $d$ is the largest distance between sources and $A$ is the minor radius of the torus. In terms of the previously defined quantities; this may be written as

$$
\frac{\mathrm{en} e}{\varepsilon_{\mathrm{o}} \mathrm{RB}_{\phi}^{2}} \mathrm{E}_{\phi}{ }^{\tau}{ }^{\tau} \tau_{2} \leqslant \mathrm{a} / \mathrm{d}
$$

for breakdown to occur in this global sense.

One may evaluate these two breakdown 11 mits 1 in terms of $E_{\phi} / P_{0}$ and $P_{0}$ to define where each is important, but to do so one must firially define $\tau_{1}$ and $\tau_{2}$. The characteristic time $\tau_{1}$ is the time it takes to disturb a freely accelerating electron from its path via collisions, either with neutrals or 1ons. The effective time for disturbing a freely accelerating electron is some hybrid between electron-ion and electron-neutral collision times. The electron-neutral collision time is given by the expression ${ }^{7}$

$$
\tau_{e-n}=3.57 \times 10^{-7} / \sigma n_{n} T_{e}^{1 / 2}
$$

The units of the parameters are $m^{-3}$ for the neutral density $n_{n}$ and $e V$ for the 
plasma temperature $\mathrm{T}_{e}$. The cross section $\sigma$ is approximately $1.21 \times 10^{-20} \mathrm{~m}^{2}$ and corresponds to a harä-sphere model of the neutral atom. There are numerous expressions for the electron-ion collision time, all of the forn

$$
\tau_{\mathrm{e}-1}=\text { constant } \cdot{ }^{\prime} \mathrm{e}^{3 / 2 / \mathrm{n}_{\mathrm{e}}}
$$

where $\mathrm{n}_{\mathrm{e}}$ is electron density in $\mathrm{m}^{-3}$. The constant varles from $9.18 \times 10^{9}$ for many sma11-angle scattering processes accumulating into a $90^{\circ}$ deflection up to $1.49 \times 10^{12}$ for a single $90^{\circ}$ coulomb collisicn. Because of the uncertainty in what atomic processes are Involved in the detalled breakdown, as well as the fact that these values for $\tau_{e-1}$ bracket the value of $\tau_{e-n}$ in a $7 \mathrm{eV}$, $3.5 \times 10^{19} \mathrm{~m}^{-3}$ electron density plasma at $50 \%$ Ionization, we will use the $\tau_{e-n}$ expression as an approximation for $\tau_{I}$. This expression is in reasonable agreement with that value computed for $\tau_{1}$ if one uses the experimental value of $\left\langle v_{\|}\right\rangle$quoted in Ref. 6. For an average temperature of $7 \mathrm{eV}(\sim 1 / 2$ the lonization potential) and $50 \%$ lonization of a 1 -mtorr fill of $\mathrm{D}_{2}, \mathrm{Eq} .(12)$ yields $\tau_{1} \simeq 318 \mathrm{~ns}$. The $50 \%$ figure us d here $1 \mathrm{~s}$ nigher than is usually used In breakdown studies, but the dependince on Ionization is falrly weak and the high figure is ised because the equilibrium loss is an MHD effect. The effect of lowering the assumed level will be discussed later.

To estimate $\tau_{2}$, note that the fielul $\left\langle E_{1}\right\rangle$ produced by the curvature drift can only be as large as the energy that creates it will allow; that is,

$$
E_{0} \frac{\left\langle E_{\perp}\right\rangle^{2}}{2}<\frac{n_{e} m_{e}}{2}\left\langle v_{\perp}\right\rangle^{2}(\vec{B} \cdot \nabla) \vec{B} \text {. }
$$

Substituting the expression for $\left\langle F_{1}\right\rangle$ from Eq. (6) we arrive at

$$
\tau_{2} \leq\left[\frac{\varepsilon_{\mathrm{o}} \mathrm{m}_{\mathrm{e}}}{2 \mathrm{n}_{\mathrm{e}} \mathrm{e}^{2}}\right] 1 / 2
$$


which is on the order of 1 ps for $50 \%$ lontzation of a 1 -mtorr $D_{2}$ fill pressure.

Putting the above expressions for $\tau_{1}$ and $\tau_{2}$ into Eqs. (9) and (11), one may solve for representative curves of $P_{0}$, critical vs $\left(E_{\phi} / P_{0}\right)_{c r i t c a l}$ for the two limits, assuming that breakdown (and completion of a charge carrying ring) is considered to occur if more than $50 \%$ tonlzation takes place; that is, it $n_{e} \approx 0.5 n_{0}$. Such a set of curves is shown in Fig. 5. The three curves labeled with symbols are for the distance d assoclated with 1, 3, or 4 preIonization sources in the $2 T-40 M$ discharge with a typical toroldal voltage of $1900 \mathrm{~V}$, which is similar to the yoltage around a single toroldal loop for 200-kA operation prior to breakdown. The solid curve with a pronounced minimum is the breakdown limit impased on the plasma due to the convection term in Eq. (3). The vertical solid curve is the diffusion limit imposed locally by the requirement that $\left.v_{\text {ionization }}=\left\langle v_{n}\right\rangle \alpha\right\rangle D_{1} / a^{2}=v_{\text {diffusion }}{ }^{8}$ All curves are calculated assuming a volume preionization so that $a=0.2 \mathrm{~m}$. The dashed curves show the result for four pretonization sources with an assumed preionized layer of only a $=0.01$ near the wall, as might result from photoelectron emission rather than photolonization of the source gas. As before, the vertical curve (dashed) is the diffusion limit. The operating region of $2 \mathrm{~T}-40 \mathrm{M}$ is shown in the dotted area. It should be noted that if a $10 \%$ lonization is used Instead of $50 \%$, the source dependent $11 \mathrm{mit}$ of $P_{0}$ only changes by a factor of 2. The local quenching limit changes by a larger amount but 1 s still orders of magnitude from experimental conditions.

The actual response of $2 \mathrm{~T}-40 \mathrm{M}$ ( $1 \mathrm{n}$ the theory) ts changes in E/P is not obvious when displayed, as In FIg. 5, because $E / P$ and $P$ are not Independent variables. If wae examines the theoretical breakdown $11 \mathrm{mit}$ for constant $E_{\phi}$ as a function of $d$, he finds tha. 'critical increases as d goes up. As is clear from Fig. 5, the operating breakdown region for ZT-40M 1 s several orders of magnitude inside the theoretical limits lmposed by the source thickness a. If a source region of only $a=0.01$ width is assuned (as might be expected for photoelectron emission from the 1iner), the breakdown limits observed are much closer to the theoretical ifmit. It is believed by the authors that the preionization is in fact a surface effect and that a source region on the order of $0.01 \mathrm{~m} 1 \mathrm{n}$ width $1 \mathrm{~s}$ indicated. The theocetical critical pressure at constant $E_{\phi}$ for three source region widths $(0.01,0.0075,0.005 \mathrm{~m}) 1 \mathrm{~s}$ plotted as a function of $d$ in Fig. 6. All regions above a particular curve are 


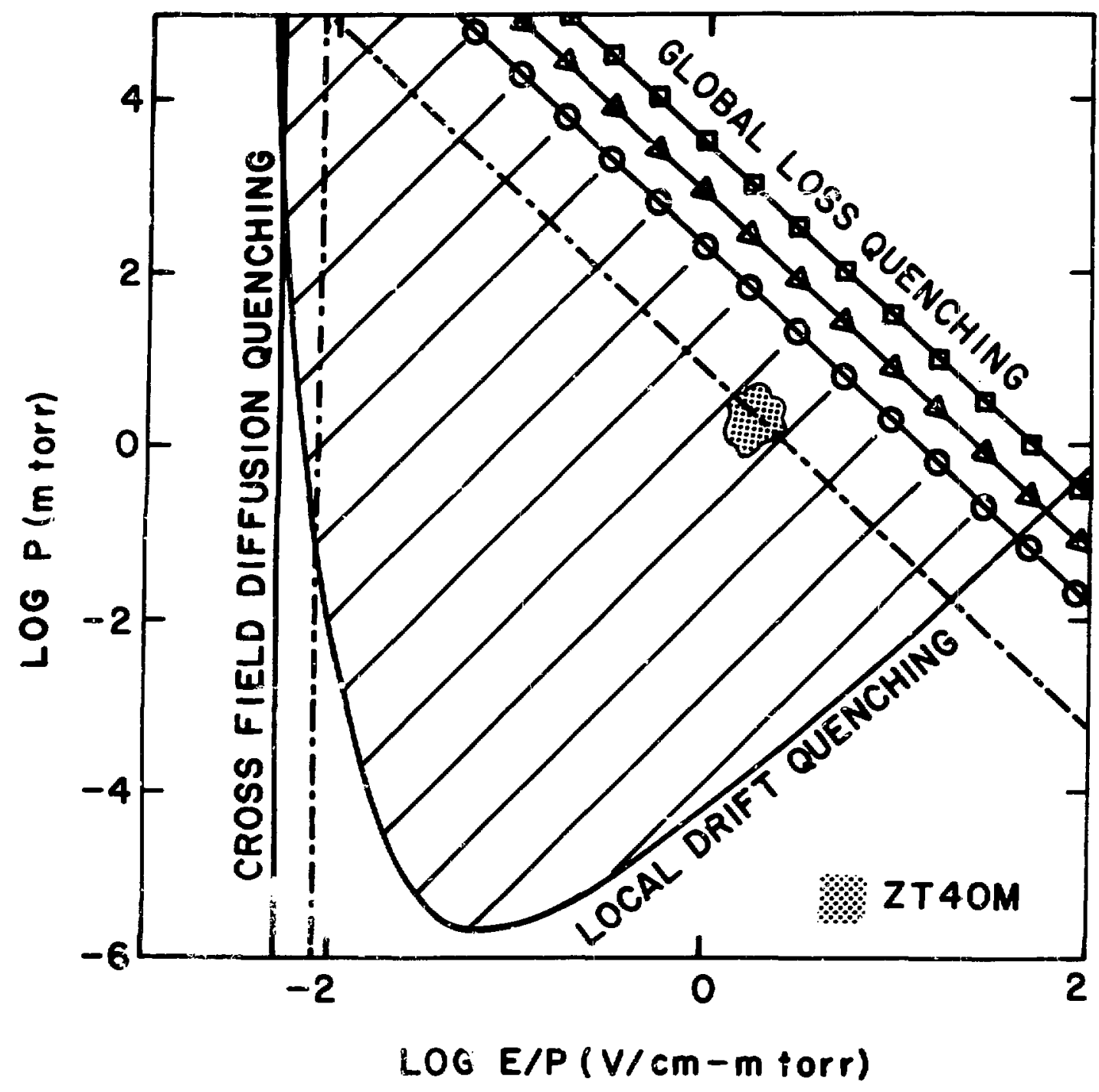

F1g. 5.

Breakdown 11mits predicted by the simple theory evaluated at $50 z$ ionization. The curves labeled with symbols correspond to vartous numbers of sources in the case of $\mathrm{ZT}-40 \mathrm{M}(O=4$ sources, $\Delta=3$ sources, $0=1$ source). Also Inciuded are the lintts due to diffusion and local quenching via the major radial drift. The dashed curves represent calculations where the source was assined to be only $1 \mathrm{cn}$ wide rather than the $20-\mathrm{cm}$ width assuned for a volume preionization source.

accessible theoretically. Also included In Fig. 6 are three experimental points that have an apparent dependence on $d$, which 18 weaker than 


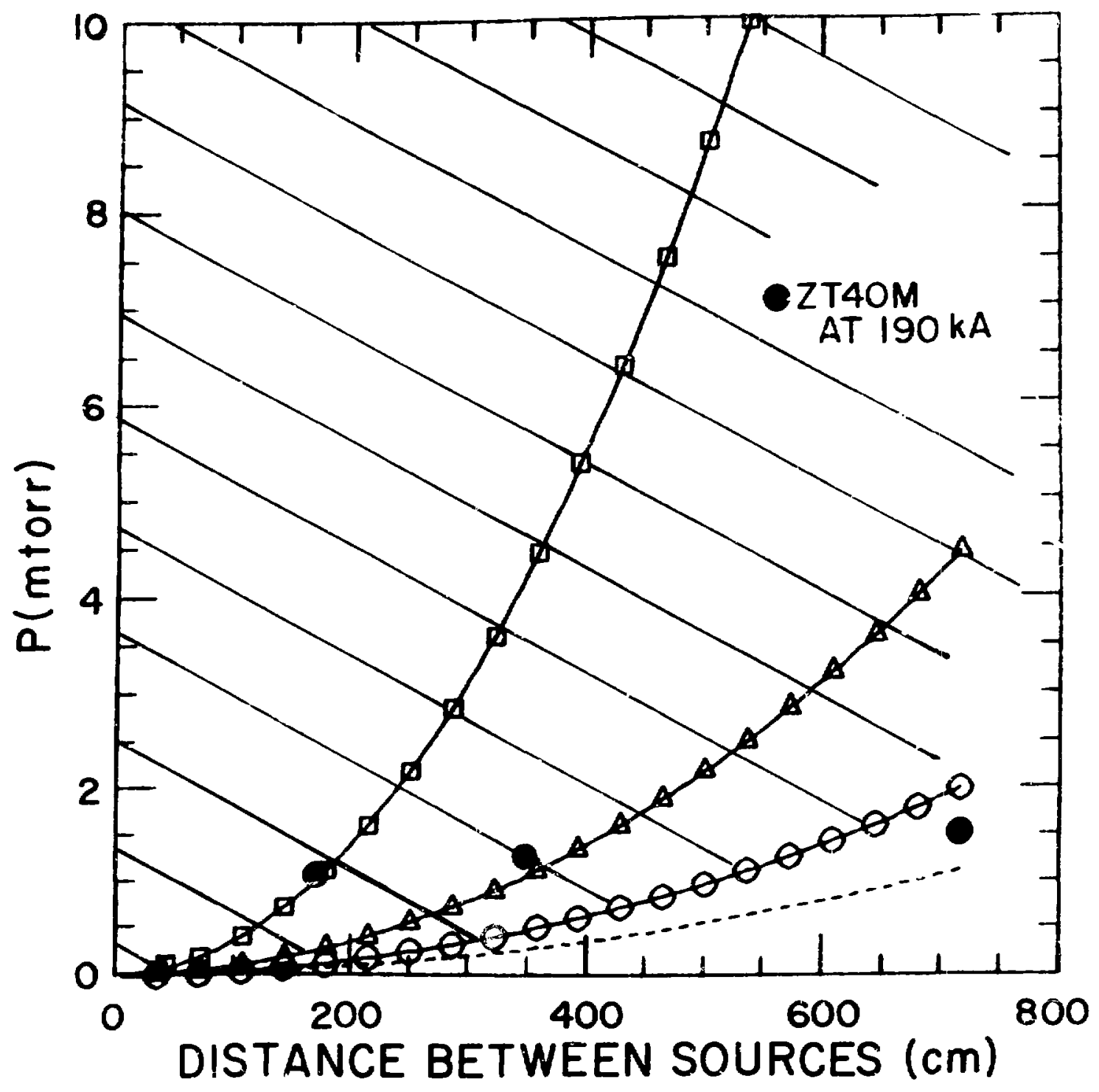

Fig. 6.

Breakdown limit as a function of distance between sources for constant $E_{\phi^{*}}$ The solid curves are for three different assumed source widths ( $0=3 \mathrm{~cm}$, $\Delta=2 \mathrm{~cm}, \square=1 \mathrm{~cm})$. The dashed curve shows the theoretical effect of doubling the inttal toroidal fleid (for a source width of $1 \mathrm{~cm}$ ). Also shown are the breakdown 11mits observed in 2T-40M for 190-kA opleration.

theoretically expected. The dashed curve In Fig. 6 shows the breakdown 1 imit predicted by the theory when twice as large an inftial toroldal field $B_{\phi}$ is 
present $(0.26 \mathrm{~T}$ compared to the experimental $0.13 \mathrm{~T}$, for a source width of $0.01 \mathrm{~m})$.

\section{v. CONCLUSIONS}

The experimental data for breakdown in ZT-40M appear to have a dependence on the number of lonization sources (up to four) present. A simple theory has been presented that is qualitatively consistent with the observation but is quantitatively quite far off. One possible explanation for the numerical disagreement has been presented that indicates the preionization source may be of very $11 \mathrm{mited}$ extent $(0.01 \mathrm{~m})$ and is believed by the authors to account for the discrepancy. This theory indicates that further gains in the breakdown IImit created by additional sources must be assoclated with decreasing distance from source to source, which is difficult beyond four sources for reasons of access to the discharge. Hidden in the formulation of the problem, however, is another dependence, $P_{O}$, critical $\propto \mathrm{F}_{\phi}^{2} / \mathrm{B}_{\phi}^{4}$, which is much more easily controlled than is the source separation, as shown In F1g. 6. A previous theory (Ref. 6), which only included the preliminary curvature and gradient $B$ driftis and did not include the hore dominant $\vec{F}_{z} \times \vec{B}_{\phi}$ drift that results from them, shows a weaker $E_{\phi} / B_{\phi}$ dependence than this. If the theory presented here is correct, further gains in ZT-40M's operating regime, if needed, may be obtainable by increasing the initial toroldal field or decreasing the loop voltage. If it is desired to probe the regions of fill pressure below the present 1 -mtorr $11 \mathrm{mlt}$, the first tests should be conducted by varying $B_{p}$ to values considerably higher than the present $0.13 \mathrm{~T}$ used for the mafority of discharges to date. (Lowering $E_{\phi}$ would also be effective but requires an additional low voltage prelonization bank.) Initlal observations at lower $B_{\phi}$ show substantial increases in $P_{0}$ consistent with the Inverse $B_{\phi}$ dependence of both theories, but neither seems to be correct in the exponent. The actual dependence seems weaker than elther theory 1mplles, but because many uncontrollable variables change along with $B_{\phi}$, absolute answers are difficult to achieve. The same statement may be applied in the case of the observed dependence of $P_{0}$ on $a$ which $1 s$ weaker than the $d^{2}$ aependence the simple theory predicts. Because the plasma appears able to control the $B_{\phi}$ field once it is fully conducting, it is possible much higher inftial flelds may be applied to aid hreakdown without harmful effects on plasma performance. 


\section{REFERENCES}

1. R. G. Watt, "Plasma Current Fluctuations and Decay Rate Behavior in the ZT-40 Device," Los Alamos National Laboratory report LA-8896-MS (July 1981).

2. N. A. Krall and A. W. Trivelplece, Princlples of Plasma Physlcs, McGrawHi11 (1973).

3. C. L. Longmire, Elementary Plasma Phys1c8, John W1ley \& Sons (1963), p. $74 \mathrm{ff}$.

4. K. Hirano, Institute of Plasma Physics report IPPJ-164, Nagoya University, Nagoya, Japan (June 1973).

5. D. J. Rose and M. Clark, Plasmas and Controlled Fus1or, M.T.T. Press (1961).

6. E. Nasser, Eundamentals of Gaseoug Ionization and Plasma Electronics, John W1.ley \& Sons $(1971)$.

7. B. Samuel Tannenbaum, Plasma Phys1cs, McGraw-H111 (1967).

8. R. Papoular, Nuc1. Fuston 18, 37 (1976). 